\title{
The Stabilizing Effect of Moisture on the Solid-State Degradation of Gabapentin
}

\author{
Zhixin Zong, ${ }^{1}$ Salil D. Desai, ${ }^{1}$ Aditya M. Kaushal, ${ }^{2}$ Dewey H. Barich, ${ }^{3}$ Hong-Shian Huang, ${ }^{1}$ Eric J. Munson, ${ }^{4}$ \\ Raj Suryanarayanan, ${ }^{2}$ and Lee E. Kirsch ${ }^{1,5,6}$
}

Received 8 December 2010; accepted 16 June 2011; published online 9 July 2011

\begin{abstract}
Gabapentin is known to undergo intramolecular cyclization to form a lactam (gaba-L) with concomitant loss of water. Gabapentin was milled in a planetary mill for 15-60 min. Unmilled and milled gabapentin were stored at $50{ }^{\circ} \mathrm{C}$ with relative humidity ranged between $5 \%$ and $90 \%$. The unmilled and milled samples were assayed for gabapentin and gaba-L by reversed phase-high-performance liquid chromatography and also subjected to powder X-ray diffraction, solid-state nuclear magnetic resonance and surface area analyses. The rates of lactamization in the milled gabapentin samples correlated to increased surface area, milling duration, and in-process lactam levels. This effect of milling could not be explained solely by the increase in surface area with increased milling time but was more likely due to increased regions of crystal disorder caused by the mechanical and thermal milling stresses. The lactamization rate of milled gabapentin samples was greatest in the presence of the lowest humidity conditions and dramatically decreased with increasing humidity. In particular, milled gabapentin appeared to be much more stable at humidity levels greater than $31 \% \mathrm{RH}$. This finding could not be attributed to the possibility of lactam hydrolysis at high humidity but rather to a competitive annealing process wherein milling-induced crystal defects were lost upon exposure to atmospheric moisture thereby stabilizing the milling-damaged drug substance.
\end{abstract}

KEY WORDS: drug degradation kinetics; gabapentin; lactamization; moisture effect; solid-state stability.

\section{INTRODUCTION}

The research reported herein was part of a multi-institutional project entitled Development of Quality by Design $(Q b D)$ Guidance Elements on Design Specifications Across Scales with Stability Considerations that encompassed fundamental physical chemical properties investigations; laboratory- and pilot-scale unit operations studies, and process simulation model development efforts conducted by researchers from eight universities over 30 months. The overarching objectives of the project were to investigate approaches to apply $\mathrm{QbD}$ principles and modelbuilding technologies to incorporate solid oral drug product stability and scale-up issues into manufacturing quality assurance. A model compound was chosen for this project based on its

\footnotetext{
${ }^{1}$ Division of Pharmaceutics, University of Iowa, Iowa City, Iowa 52242, USA.

${ }^{2}$ Department of Pharmaceutics, University of Minnesota, Minneapolis, Minnesota 55455, USA.

${ }^{3}$ Department of Pharmaceutical Chemistry, University of Kansas, Lawrence, Kansas 66047, USA.

${ }^{4}$ Department Pharmaceutical Sciences, University of Kentucky, Lexington, Kentucky 40536-0596, USA.

${ }^{5}$ College of Pharmacy, University of Iowa, Iowa City, Iowa 52242, USA.

${ }^{6}$ To whom correspondence should be addressed. (e-mail: lee-kirsch@uiowa.edu)
}

known propensity to undergo physical and chemical transformations and its current use as a solid oral drug product. This report is one of a series of the fundamental physical chemical properties investigations and, in fact, represents the first in a number of reports on linkage between physical and chemical instability of the model compound.

Gabapentin is a GABA analogue used for the treatment of epilepsy and neuropathic pain. This $\gamma$-amino acid has been reported to exist as three polymorphs and a monohydrate and the crystal structures of all of these forms have been reported (1-3). The commercial drug substance is form II ( $\alpha$-gabapentin). Form III ( $\beta$-gabapentin) has been crystallized from a saturated ethanol solution with heat $\left(60^{\circ} \mathrm{C} ; 3\right)$. The form IV ( $\gamma$-polymorph) was similarly prepared but at room temperature. Interestingly, form III is reportedly unique in that intramolecular hydrogen bonding occurs between the amine and carboxylic acid groups whereas only intermolecular hydrogen bonding has been associated with the crystalline structures of forms II and IV between these groups in the $\alpha$ and $\beta$ forms. Form I is the hydrate (3).

The interconversion between the polymorphic forms has been reported to occur by thermal treatment and milling (4-6). In the latter case, the presence of various excipients has been reported to induce differences in the polymorphic composition of co-milled gabapentin (6).

In solution, gabapentin can exist in four ionic forms: cationic, zwitterionic, neutral, and anionic species. The carbox- 
ylate and amine pKa have been reported to be 3.7 and 10 , respectively (7-9).

Three studies have been reported on the kinetics and mechanisms of gabapentin instability at elevated temperatures in aqueous solution (7-9). Gabapentin degrades by intramolecular cyclization to form a five-membered lactam by nucleophilic attack of the alkyl amine on the carboxylate carbonyl followed by dehydration (Scheme 1). This lactamization reaction is $\mathrm{pH}$-dependent with minimum rate at $\mathrm{pH}$ 5.5-6 and is buffer catalyzed. It has been hypothesized that the reactive form is the neutral species which is in equilibrium with the predominant zwitterionic form and requires proton transfer from the amine to the carboxylate.

In lyophilized samples of gabapentin in the presence of various excipients (PVP K30, calcium sulfate, HP- $\beta-C D$, SBE$\beta-\mathrm{CD}$, mannitol, D-raffinose, trehalose and lactose), lactamization was studied upon storage at $50^{\circ} \mathrm{C}$ and various relative humidity conditions $(0 \%, 45 \%$, and $75 \% ; 10)$. The rate of lactamization was highest in the presence of cyclodextrins and PVP K30 and lowest in the neat sample. Lactose also appeared to increase lactamization. High humidity did not consistently increase lactamization rates. Accelerated lactamization in the presence of cyclodextrin (perhaps associated with the formation of inclusion complexes) have been reported in aqueous solution as well as solid-state $(7,10)$.

Minimizing lactamization is a critical product quality attribute in that gabapentin lactam has been reported to be 20-fold more toxic (9). Thus, the United States Pharmacopeia (USP) limit on lactam is less than $0.4 \%$. A number of patents have been issued for stable solid and liquid formulations and manufacturing processes to achieve this target (11-18). The stability of split and intact tablets have also been compared and found to be the same $(19,20)$.

The key objective of the work presented here with respect to the overarching project goals was to (1) provide evidence for the linkage between manufacturing-stress induced physical change and subsequent chemical instability for the model compound, gabapentin, and (2) provide a rational explanation for the unexpected relation between chemical instability and atmospheric humidity during storage. The first objective was an essential requirement for the project's success whereas the latter objective was necessary first step in the development of a useful solid-state drug degradation model.

\section{MATERIALS AND METHODS}

Gabapentin was obtained from Hangzhou Starshine Pharmaceutical Co. LTD (Hangzhou, China) and gabapentin lactam (gaba-L) was purchased from Sigma-Aldrich (St. Louis, MO, USA).

Gabapentin is known to undergo intramolecular cyclization to form a gaba-L with concomitant loss of water; however, the
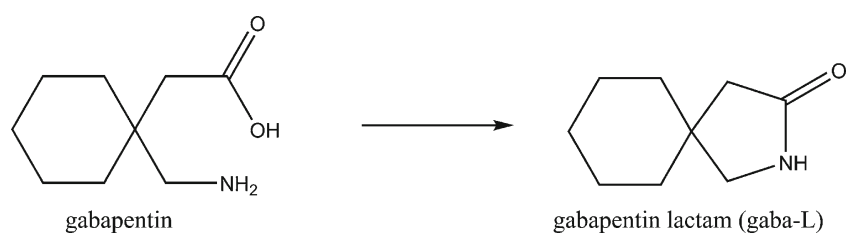

Scheme 1. Lactamization of gabapentin drug substance itself (crystalline form II anhydrate) is resistant to chemical instability. In order to generate relatively unstable gabapentin and to evaluate the effects of manufacturing stress on chemical stability, approximately $3 \mathrm{~g}$ of drug substance was placed in a 45-mL milling bowl with four hardened steel balls $(15 \mathrm{~mm})$ and milled in a planetary mill (Pulviserette 7, Planetary Micro Mill) for 15-60 min. Unmilled and milled gabapentin were stored at $50^{\circ} \mathrm{C}$, and the relative humidity ranged between $5 \%$ and $80 \%$ using storage chambers containing saturated salt solutions as described below. The unmilled and milled samples were assayed for gabapentin and gaba-L by reversed phase-highperformance liquid chromatography (HPLC) and also subjected to powder X-ray diffraction (PXRD), solid-state nuclear magnetic resonance (NMR), and surface area analyses.

\section{ANALYTICAL METHODS}

\section{Chromatographic Methods}

Chemical instability measurements were made using two methods both of which involved detection of the $\%$ mole formation of gaba-L by chromatographic methods. (1) The level of gaba-L generated during milling was determined using samples taken before and after milling and measuring gaba-L levels by HPLC methods described below. (2) The susceptibility of process-stressed gabapentin to thermally induced chemical degradation was determined by storing an aliquot of milled gabapentin at $50^{\circ} \mathrm{C}(-5 \% \mathrm{RH})$ for $24 \mathrm{~h}$. The initial rate of gaba$\mathrm{L}$ formation (i.e., lactamization rate) was determined by measuring the time-dependent increase in \% mole concentration of gaba-L in the thermally treated solid after 24-h storage.

Chromatographic analysis was carried using a high/low assay (21) wherein trace amounts of degradation product (gaba-L) were measured using high concentrations of parent gabapentin $(\sim 12 \mathrm{mg} / \mathrm{mL})$ and gabapentin mass was measured at low concentations (about $2.4 \mathrm{mg} / \mathrm{mL}$ ). These two results were then used to compute the gaba-L content as a mole percentage $(w / w)$ of total gabapentin (gabapentin plus gaba-L). Calibration standards for the gaba-L and gabapentin were prepared in the range of $0.0005-0.005 \mathrm{mg} / \mathrm{mL}$ and $0.5-5 \mathrm{mg} / \mathrm{mL}$, respectively. The HPLC system was a Thermo Spectrum System P4000 pump, AS3000 auto injector, and UV 6000 LP photodiode array detection system. The column was $\mu$ Bondapak Cyano column 3.9 x 300mm. Isocratic analysis was conducted using a mobile phase composed of 95 parts buffer $\left(10 \mathrm{mM} \mathrm{KH} \mathrm{KO}_{4} / 10 \mathrm{mM} \mathrm{K} \mathrm{K}_{2} \mathrm{HPO}_{4}\right)$ and five parts acetonitrile. Mobile phase was filtered through a $0.2-\mu \mathrm{m}$ filter. Analysis was carried out using an analytical wavelength of $210 \mathrm{~nm}$, flow rate of $1.0 \mathrm{~mL} / \mathrm{min}$, and a $0.020 \mathrm{~mL}$ injection volume. Run times were $10 \mathrm{~min}$. Retention volumes for gabapentin and gaba-L were 3.8 and $7.6 \mathrm{~mL}$, respectively (Fig. 1). Calibration plots for gabapentin and gaba-L were linear at the range of $0.5-10 \mathrm{mg} / \mathrm{mL}$ and $0.5-10 \mu \mathrm{g} / \mathrm{mL}$.

\section{Surface Area Measurement}

Milled and unmilled aliquots of gabapentin were evaluated by measuring the specific surface areas by nitrogen adsorption. To fractionate gabapentin into different particle sizes ranges, a $5 \mathrm{~g}$ aliquot of gabapentin was placed in \#35 sieve on top of a six sieves of various sizes. Then all sieves 


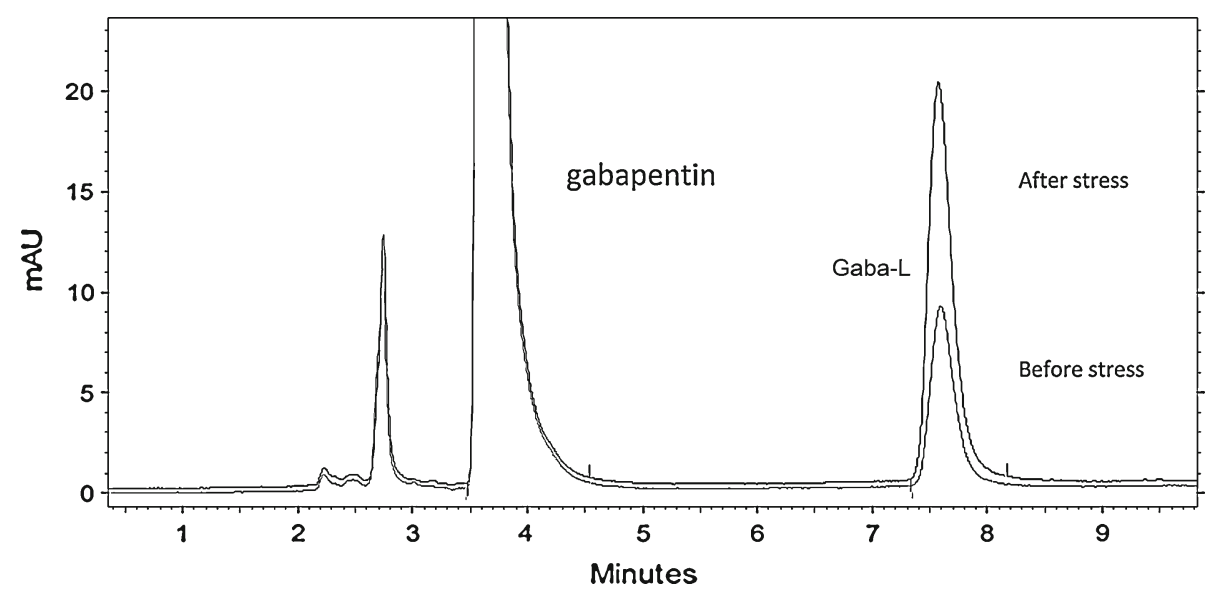

Fig. 1. Representative HPLC chromatograms showing peaks for gabapentin and gaba-L. Thermal stress condition was $50^{\circ} \mathrm{C}$ in a sealed vial for 1 day

were loaded in a Vibratory 3-in Sieve Shaker (Model SS-5, Gilbson Company, Inc, Worthington, $\mathrm{OH}$ ). After shaking for $0.5 \mathrm{~h}$, powder particles were distributed into sieve fractions using the following sieves: \#35 (500 $\mu)$, \#120 (125 $\mu)$, \#140

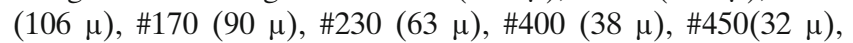
$\# 500(27 \mu)$, and \#635 (20 $\mu)$.

Specific surface area was determined using a Quantasorb sorption system. Nitrogen was used as adsorbate gas and helium was used as carrier gas. A five-point BET analysis was conducted on the samples and the specific surface area was calculated based on BET plot and weight of samples.

\section{TGA and DSC}

A thermo-gravimetric analyzer (TGA, Model Q50, TA Instruments, New Castle, DE, USA) and a differential scanning calorimeter (DSC, Model 2920, TA Instruments) were connected to a thermal analysis operating system (Thermal Analyst 2000, TA Instruments). Approximately 2$6 \mathrm{mg}$ of the sample, in a crimped aluminum pan or an open aluminum pan, was heated from room temperature to $250^{\circ} \mathrm{C}$ at varying rates under nitrogen purge.

\section{Powder X-ray Diffraction}

XRD analysis was conducted as follows: samples were filled in a glass holder and exposed to $\mathrm{Cu} \mathrm{K \alpha}$ radiation $(45 \mathrm{kV} \times 40 \mathrm{~mA})$ in a wide angle X-ray diffractometer (model D5005, Bruker, Madison, WI, USA) at ambient temperature. The instrument was operated in a step-scan mode, in $0.05^{\circ} 2 \theta$ increments, and counts were accumulated for $1.0 \mathrm{~s}$ at each step over the angular range of $5-40^{\circ} 2 \theta$. Data analyses were performed with commercially available software (JADE, version 8.0, Materials Data Inc., Livermore, CA, USA).

\section{Solid-state NMR}

Solid-state NMR spectra were acquired on a CMX spectrometer operating at $300 \mathrm{MHz}$ for proton and $75 \mathrm{MHz}$ for carbon. Samples were packed into a Revolution NMR $7 \mathrm{~mm}$ (o.d.) zirconia rotor. The spectra were acquired with cross polarization $(\mathrm{CP})$ using a linear ramp on the proton channel during the $\mathrm{CP}$ period. The optimal contact time varied between samples but was typically around $0.9 \mathrm{~ms}$. Magic angle spinning was done at a rate of $4.0 \mathrm{kHz}$. Proton spin-lattice relaxation data were acquired via saturation recovery. Spinning sidebands were eliminated from nonrelaxation spectra via sideband suppression techniques.

\section{Solid-State Degradation}

To evaluate the susceptibility of gabapentin materials to thermally-induced degradation, an aliquot of gabapentin (approximately $15 \mathrm{mg}$ ) was weighed and placed in a USP type II glass vial, wrapped with aluminum foil, and then stored in desiccators containing anhydrous calcium sulfate (Drierite $\AA$ ) or saturated salt solutions to control the relative humidity at the following values $\left(50^{\circ} \mathrm{C}\right) 5$ (Drierite), 30.5 $\left(\mathrm{MgCl}_{2}\right), 46.9\left(\mathrm{Mg}\left[\mathrm{NO}_{3}\right]_{2}\right), 74.4(\mathrm{NaCl})$, and $81.2(\mathrm{KCl}) \% \mathrm{RH}$ at $-50^{\circ} \mathrm{C}$ for $24,48,72$, and $96 \mathrm{~h}$.

Periodically, a $1.0-\mathrm{mL}$ aliquot of mobile phase was injected into a sample vial containing thermally stress gabapentin by using a $25 \mathrm{G}$ needle and $1.0-\mathrm{mL}$ syringe. The vials were shaken until the sample dissolved. Solutions were assayed for gaba-L (high concentration) and diluted fivefold (low concentration) with mobile phase for analysis of gabapentin. The gaba-L concentration was calculated as a molar percentage of gabapentin.

\section{Solution Degradation}

Thirty $\mathrm{mM}$ gaba-L solutions were prepared in $\mathrm{pH} 2$ and 7 $0.1 \mathrm{M}$ phosphate buffer and $\mathrm{pH} 8.50 .1 \mathrm{M}$ borate buffer and stored in type II glass vials, sealed with a teflon-faced butyl rubber stopper and crimp-secured with an aluminum seal. Vials were immersed in $80^{\circ} \mathrm{C}$ water bath for 5 days. A $0.5-\mathrm{mL}$ aliquot was taken by using $25 \mathrm{G}$ needle and $1.0-\mathrm{mL}$ syringe at different time points and subjected to HPLC assay for gabapentin and gaba-L.

\section{RESULTS}

Representative HPLC chromatograms for samples of gabapentin before and after thermal stress $\left(50^{\circ} \mathrm{C}, 5 \% \mathrm{RH}\right)$ for $24 \mathrm{~h}$ are depicted in Fig. 1. Retention volumes for gabapentin and lactam were 3.8 and $7.6 \mathrm{~mL}$, respectively. 
Table I. The Accuracy of HPLC Method

\begin{tabular}{|c|c|c|c|c|c|c|c|c|c|}
\hline \multirow[b]{2}{*}{ Sample ID } & \multirow{2}{*}{$\begin{array}{l}\text { weight of } \\
\text { Gabapentin (mg) }\end{array}$} & \multicolumn{2}{|c|}{ Gabapentin detected } & \multicolumn{2}{|c|}{ Gaba-L added } & \multicolumn{2}{|c|}{ Gaba-L detected } & \multicolumn{2}{|c|}{ Detected addition of Gaba-L } \\
\hline & & Weight (mg) & Recovery (\%) & Weight $(\mu \mathrm{g})$ & Mole $\%$ & Weight $(\mu \mathrm{g})$ & Mole\% & Mole \% & Recovery (\%) \\
\hline o1 & 14.51 & 14.34 & 98.82 & 0 & & 1.056 & 0.008 & & \\
\hline $\mathrm{o} 2$ & 12.43 & 12.41 & 99.81 & 0 & & 0.761 & 0.007 & & \\
\hline 03 & 11.99 & 11.86 & 98.91 & 0 & & 0.765 & 0.007 & & \\
\hline A1 & 11.42 & 11.40 & 99.81 & 1.0212 & 0.010 & 1.813 & 0.018 & 0.010 & 103.30 \\
\hline $\mathrm{A} 2$ & 13.3 & 13.47 & 101.25 & 1.0212 & 0.008 & 1.919 & 0.016 & 0.008 & 100.18 \\
\hline A 3 & 11.06 & 11.22 & 101.49 & 1.0212 & 0.010 & 1.830 & 0.018 & 0.011 & 106.11 \\
\hline B1 & 11.93 & 12.11 & 101.50 & 2.553 & 0.024 & 3.343 & 0.031 & 0.023 & 99.40 \\
\hline B2 & 13.49 & 13.41 & 99.37 & 2.553 & 0.021 & 3.591 & 0.030 & 0.022 & 105.73 \\
\hline B3 & 13.41 & 13.51 & 100.72 & 2.553 & 0.021 & 3.477 & 0.029 & 0.021 & 101.00 \\
\hline $\mathrm{C} 1$ & 14.4 & 13.96 & 96.96 & 5.106 & 0.041 & 5.971 & 0.048 & 0.040 & 98.74 \\
\hline $\mathrm{C} 2$ & 11.81 & 12.01 & 101.68 & 5.106 & 0.048 & 6.193 & 0.058 & 0.050 & 105.63 \\
\hline C3 & 14.28 & 13.96 & 97.74 & 5.106 & 0.041 & 6.179 & 0.049 & 0.042 & 102.82 \\
\hline D1 & 12.11 & 12.05 & 99.52 & 10.212 & 0.095 & 11.063 & 0.102 & 0.095 & 100.47 \\
\hline $\mathrm{D} 2$ & 12.34 & 12.33 & 99.94 & 10.212 & 0.092 & 10.882 & 0.099 & 0.091 & 98.52 \\
\hline D3 & 12.48 & 12.60 & 100.99 & 10.212 & 0.090 & 11.098 & 0.098 & 0.091 & 100.45 \\
\hline E1 & 12.92 & 12.68 & 98.16 & 25.53 & 0.224 & 26.045 & 0.229 & 0.222 & 98.70 \\
\hline E2 & 11.98 & 12.03 & 100.39 & 25.53 & 0.237 & 26.136 & 0.242 & 0.235 & 99.23 \\
\hline E3 & 11.17 & 11.15 & 99.80 & 25.53 & 0.255 & 26.259 & 0.263 & 0.255 & 99.94 \\
\hline F1 & 10.86 & 11.13 & 102.51 & 51.06 & 0.510 & 51.439 & 0.514 & 0.506 & 99.28 \\
\hline $\mathrm{F} 2$ & 13.48 & 13.45 & 99.79 & 51.06 & 0.422 & 52.273 & 0.432 & 0.425 & 100.61 \\
\hline F3 & 10.72 & 10.80 & 100.78 & 51.06 & 0.525 & 51.826 & 0.533 & 0.526 & 100.08 \\
\hline G1 & 12.58 & 12.49 & 99.28 & 127.65 & 1.129 & 127.370 & 1.127 & 1.119 & 99.13 \\
\hline $\mathrm{G} 2$ & 11.2 & 11.26 & 100.51 & 127.65 & 1.251 & 128.290 & 1.258 & 1.250 & 99.90 \\
\hline G3 & 13.04 & 12.91 & 99.02 & 127.65 & 1.093 & 127.901 & 1.095 & 1.088 & 99.51 \\
\hline
\end{tabular}

Calibration plots for gabapentin and lactam were linear at the range of $0.5-10 \mathrm{mg} / \mathrm{mL}$ and $0.5-10 \mu \mathrm{g} / \mathrm{mL}$. Upon thermal stress $\left(50^{\circ} \mathrm{C}, 24 \mathrm{~h}, 5 \% \mathrm{RH}\right)$, no significant decrease in gabapentin peak area was observed for this sample; however, the gaba-L peak area approximately doubled which illustrates the sensitivity of the HPLC method to very small changes in gaba-L concentration. The limit of detection for gaba-L was determined by the signal to noise ratio of $3-0.1 \mu \mathrm{g} / \mathrm{mL}$, and the relative standard deviation for multiple analyses (six sequential injections of a $0.1 \mu \mathrm{g} / \mathrm{mL}$ gaba-L solution) was $9.5 \%$. The limit of quantification of gaba-L was determined by the signal to noise ratio of 10 was $0.5 \mu \mathrm{g} / \mathrm{mL}$ and the RSD for six injections of $0.5 \mu \mathrm{g} / \mathrm{mL}$ gaba-L solution was $1.4 \%$ (Table I).

Milling was used to induce manufacturing-type stress in gabapentin. The effects of milling were evaluated by surface area determination, PXRD, solid-state NMR, and chemical instability. The specific area increased from 0.20 to $17.7 \mathrm{~m}^{2} / \mathrm{g}$ with milling duration of 0-60 min (Table II). Unmilled and milled gabapentin samples showed similar PXRD patterns (Fig. 2) that were consistent with maintenance of form II as the predominant polymorphic form, although the intensity of typical form II XRD peaks at $7.8^{\circ}$ and $14.9^{\circ} 2 \theta$ decreased with increased milling time. Solid-state NMR spectra (Fig. 3) showed no significant changes in the chemical shifts for milled and unmilled samples although again significant peak broadening was observed.

The effects of milling on chemical stability was measured in two ways: the change in gaba-L content before and after milling ("in-process lactam") and the susceptibility of milled samples to further degradation (rate of lactimization at $50^{\circ} \mathrm{C}$ ).

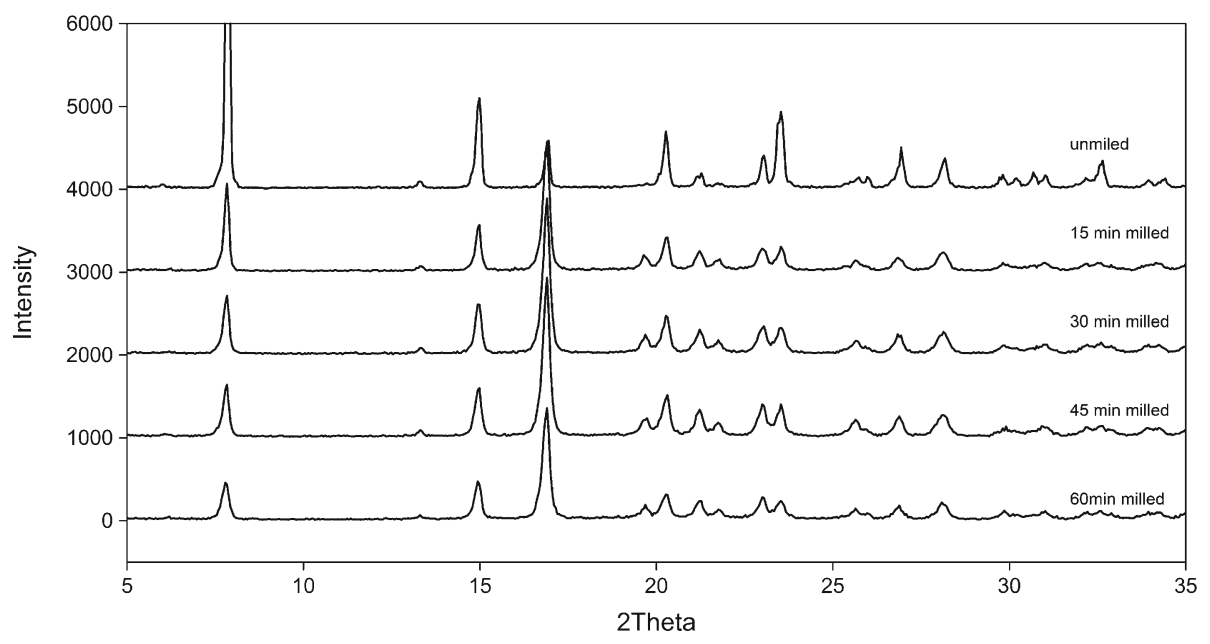

Fig. 2. XRD patterns of unmilled and milled gabapentin samples 


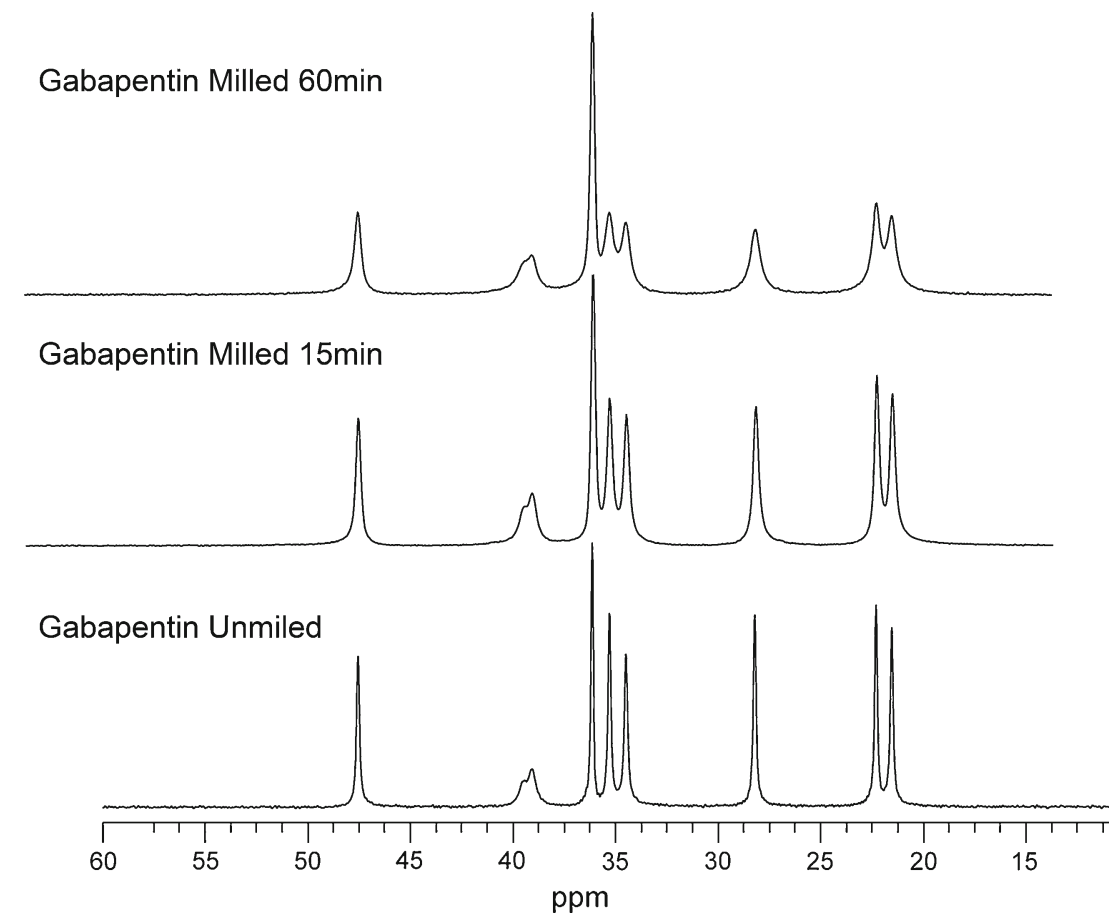

Fig. 3. Solid-state NMR spectrum of unmilled and milled gabapentin samples

During the milling operation gaba-L levels increased from $0.007 \mathrm{~mol} \%$ for unmilled gabapentin to $0.042,0.095,0.152$, and $0.234 \mathrm{~mol} \%$ during milling for $15,30,45$, and $60 \mathrm{~min}$, respectively.

Milled samples were subsequently thermally stressed at $50^{\circ} \mathrm{C}$ and $5 \% \mathrm{RH}$ and the initial rates of lactamization were measured after $24 \mathrm{~h}$. The lactamization rate increased with increased duration of milling. The lactimization rates were 500 times faster in samples of gabapentin milled for $60 \mathrm{~min}$ compared to the unmilled samples (Table I). Moreover, increased rates of lactamization in the milled samples correlated to increased surface area, milling duration, and in-process lactam levels.

An attempt was made to investigate the relationship between surface area and lactamization rate using both milled and unmilled gabapentin by separating an aliquot of gabapentin into sieve fractions and subjecting each fraction to

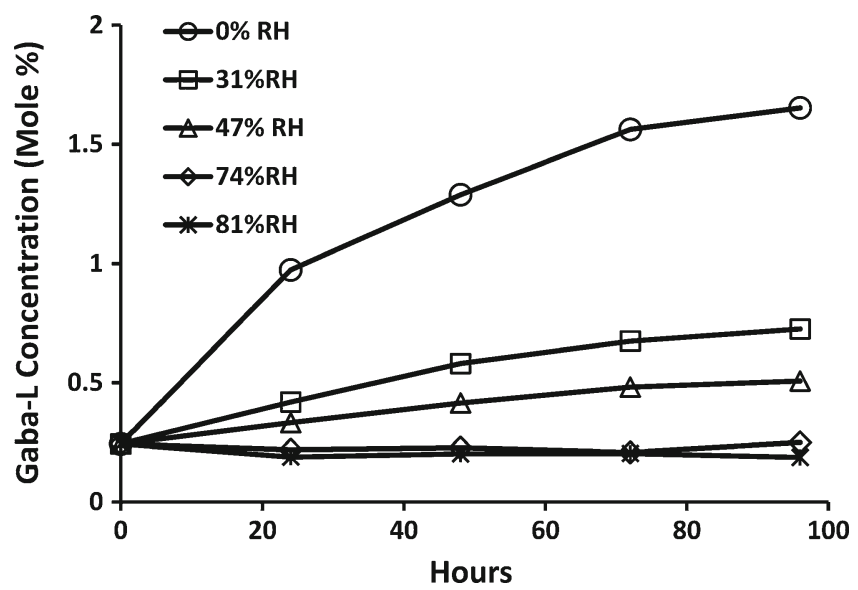

Fig. 4. Gaba-L formation from $60 \mathrm{~min}$ milled gabapentin sample at $50^{\circ} \mathrm{C}$ and different relative humidity thermal stress to determine the initial lactamization rate associated with unmilled gabapentin with different particle sizes. For the sieve fractions of unmilled gabapentin ranging in specific surface area from 0.1 to $0.9 \mathrm{~m}^{2} / \mathrm{g}$, the lactamization rate increased with increasing surface area from about $0.007-0.015 \mathrm{~mol} \% / \mathrm{day}$; and for $15 \mathrm{~min}$ milled gabapentin ranging in specific surface area from 4.2 to $5.7 \mathrm{~m}^{2} / \mathrm{g}$, the lactamization rate increased from about 0.055-0.063 mol\%/day (Table II).

The rate of lactamization in milled samples exposed to different levels of atmospheric humidity at $50^{\circ} \mathrm{C}$ gave rise to the most surprising results (Fig. 4). The lactamization rate was greatest in the presence of the lowest humidity conditions and dramatically decreased with increasing humidity. In particular, milled gabapentin appeared to be much more stable at humidity levels greater than $31 \% \mathrm{RH}$.

\section{DISCUSSION}

The measurement of lactamization rate was a useful method for determining gabapentin chemical instability. One potential complication in using gaba-L appearance rate as a measure of gabapentin instability is that gaba-L is demonstrably volatile at elevated temperatures. However, the rate of evaporative loss of gaba-L was $<3 \%$ /day at $50^{\circ} \mathrm{C}$. Moreover when an aliquot of gaba- $\mathrm{L}$ was placed in a controlled humidity chamber $(5 \%, 31 \%, 47 \%, 74 \%$, or $81 \% \mathrm{RH})$ and stored for 3 days at $50^{\circ} \mathrm{C}$, the percentage recovery was $99-102 \%$. Therefore, gaba-L loss by vaporization during exposure to elevated temperatures was not expected to significantly affect the measured lactamization rate.

The lack of changes in PXRD patterns and solid-state NMR chemical shifts between milled and unmilled samples that could be attributed to polymorphic transition suggests that the extent of any milling-induced form changes under the 
Table II. Specific Surface Area, Initial Gaba-L Concentration, and Lactamization Rate

\begin{tabular}{lcccc}
\hline Milling time (minute) & $\begin{array}{l}\text { Sieve fraction (sieve } \\
\text { mesh size, microns) }\end{array}$ & $\begin{array}{l}\text { Specific surface } \\
\text { area }\left(\mathrm{m}^{2} / \mathrm{g}\right)\end{array}$ & $\begin{array}{l}\text { Lactamization } \\
\text { rate }(\mathrm{mole} \% / \text { day) }\end{array}$ \\
\hline 0 & 125 & 0.1 & 0.0037 & 0.0064 \\
0 & 106 & 0.3 & 0.0096 & 0.007 \\
0 & 90 & 0.4 & 0.0057 & 0.006 \\
0 & 63 & 0.6 & 0.0117 & 0.013 \\
0 & 38 & 0.9 & 0.055 & 0.015 \\
10 & 32 & 4.2 & 0.050 & 0.242 \\
10 & 27 & 4.7 & 0.057 & 0.245 \\
10 & 20 & 4.9 & 0.063 & 0.248 \\
10 & $<20$ & 5.4 & 0.007 & 0.250 \\
0 & & 0.2 & 0.042 & 0.002 \\
15 & & 5.7 & 0.095 & 0.355 \\
30 & & 12.1 & 0.152 & 0.632 \\
60 & & 16.2 & 0.234 & 0.842 \\
\hline
\end{tabular}

Lactamization rate was measured at $5 \% \mathrm{RH}$ and $50^{\circ} \mathrm{C}$

conditions used in our studies were sufficiently small as to avoid detection by these techniques. Additional NMR analysis on milled samples has demonstrated significant ${ }^{1} \mathrm{H}$ relaxation time changes; these results are presented in a separate manuscript. Moreover, subsequent milling studies under more rigorous conditions have revealed polymorphic changes and a separate manuscript has been prepared to discuss these results. Moreover, Lin et al. has reported polymorphic changes detected by FTIR upon more rigorous milling conditions in the presence and absence of co-milled excipients (7).

\section{Effects of Milling}

Duration of milling clearly impacted both the generation of gaba-L during this unit operation and also affected the susceptibility of the milled material for further degradation by intramolecular cyclization to form gaba-L. The effects of milling could be due to the dramatic increase in surface area associated with particle size reduction. Alternatively, crystal disorder associated with the milling stresses could also be a major factor in the increased lactamization rate.

An attempt was made to investigate the relationship between surface area and lactamization rate by separating aliquots of milled and unmilled gabapentin into sieve fractions and subjecting each sieve fraction to thermal stress to determine the dependence of lactamization rate on particle size. The magnitude of the effects of particle size and lactamization rate were compared for aliquots of gabapentin that were milled for $15,30,45$, and $60 \mathrm{~min}$ and for sieve fractions obtained from an unmilled aliquot of gabapentin or an aliquot milled for $15 \mathrm{~min}$.

For sieved and unsieved aliquots, particle size differences were measured by specific surface area. The lactamization rates are compared to the measured specific surface areas of all aliquot in Fig. 5. A least squares correlation line for each group was estimated to illustrate the magnitude of the apparent correlation between lactamization rate and specific surface area. The slope of this correlation line was five times greater for the milled unsieved aliquots than either the sieve fractions obtained from milled or unmilled aliquot. This observation suggests that the effect of milling could not be explained solely by the increase in surface area with increased milling time but was more likely due to increased regions of crystal disorder caused by the milling stresses.

\section{Effects of Moisture}

The effect of moisture on the solid-state stability of milled gabapentin was unexpected. At high humidity $(\geq 74 \%$ $\mathrm{RH}$ and $50^{\circ} \mathrm{C}$ ), the rate of lactimization was essentially zero whereas at low humidity $\left(5 \% \mathrm{RH}\right.$ and $\left.50^{\circ} \mathrm{C}\right)$, the initial rate of lactamization for the same aliquot of milled gabapentin was about $0.7 \mathrm{~mol} \% /$ day. This apparent stabilizing effect of humidity on gabapentin degradation could be explained by a number of phenomena including: (1) loss of lactam during humidity-facilitated vaporization, (2) decreased rate of lactamization due to mass law effect, (3) decreased rate of lactamization due to formation of stable gabapentin hydrate, (4) decreased rate of lactamization due to competitive crystal defect annealing process that is facilitated by surface adsorbed moisture. We have attempted to investigate each of these possibilities.

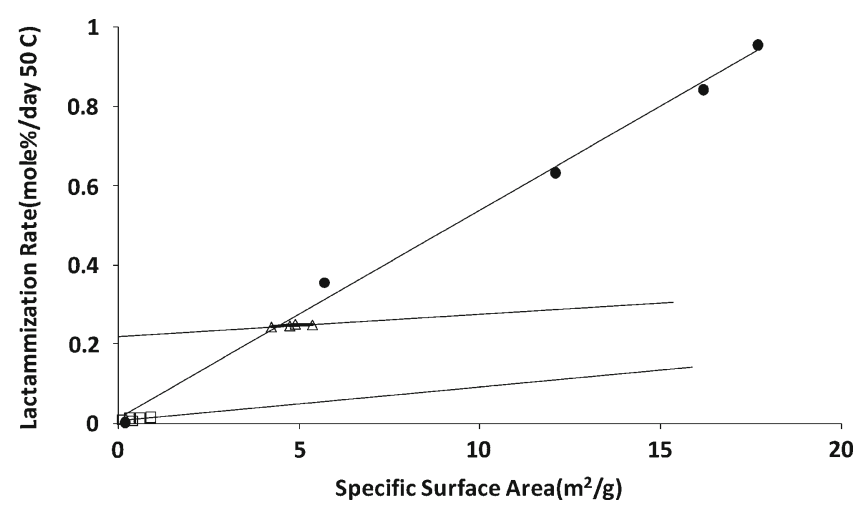

Fig. 5. Lactamization rate (mole $\% /$ day at $5 \% \mathrm{RH}$ and $50^{\circ} \mathrm{C}$ ) for gabapentin samples as a function of the measured specific surface area. Empty square sieved fractionated unmilled gabapentin, empty triangle sieved fractionated $10 \mathrm{~min}$ milled gabapentin, filled circle 15 , 30, 45, and 60 min milled gabapentin samples 
Table III. Gaba-L Recovery in Saturated Salt Solutions

\begin{tabular}{llllcc}
\hline & & & & \multicolumn{2}{c}{$\%$ Gaba-L recovered After $96 \mathrm{~h}$ at $50^{\circ} \mathrm{C}$} \\
\cline { 5 - 6 } Powders & $\mathrm{RH}(\%)$ & Saturated salt solution & Total Gaba-L mole \% & In solid & \multicolumn{2}{c}{ In saturated solution } \\
\hline Milled gabapentin & 31 & $\mathrm{MgCl}_{2}$ & 1.49 & 98.14 & 0.93 \\
& 47 & $\mathrm{Mg}\left(\mathrm{NO}_{3}\right)_{2}$ & 1.27 & 98.82 & 0.78 \\
& 74 & $\mathrm{NaCl}$ & 1.04 & 95.61 & 3.92 \\
Milled Gaba-L & 81 & $\mathrm{KCl}$ & 1.02 & 95.54 & 0.19 \\
& 31 & $\mathrm{MgCl} 2$ & 100.00 & 99.78 & 0.02 \\
& 47 & $\mathrm{Mg}(\mathrm{NO} 3) 2$ & 100.00 & 99.96 & 0.08 \\
& 74 & $\mathrm{NaCl}$ & 100.00 & 99.90 & 0.12 \\
\hline
\end{tabular}

If the rate of lactamization was same or greater in humid conditions than in a dry atmosphere, then it is conceivable that the observed rate decrease under humid conditions was due to loss of gaba-L by vaporization and subsequent dissolution of gaseous gaba-L into the saturated salt solution. This explanation is unlikely because although gaba-L is volatile, the rate of gaba-L loss by TGA is much slower than the rate of lactamization under dry conditions. Moreover, there was no apparent increase in gaba-L mass loss in the presence of saturated salt solutions and the detected amount of gaba- $\mathrm{L}$ in the saturated salt solutions used to humidify the storage chamber was $\leq 5 \%$ of the amount of gaba-L present in the solid state (Table III).

Intramolecular lactamization of gabapentin is a dehydration reaction resulting in the stoichiometric formation of gaba- $\mathrm{L}$ and water. Therefore, if the reaction is reversible, then the presence of surface-adsorbed water could decrease the lactamization rate by mass law effect. This hypothesis was tested using two experiments. Firstly, an aliquot of milled gaba-L powder was subjected to elevated temperature $\left(50^{\circ} \mathrm{C}\right)$ and humidity conditions $(31-81 \% \mathrm{RH})$ for 3 days, and the gaba-L mass balance and the appearance of gabapentin was determined by HPLC. Secondly, an attempt was made to hydrolyze gaba-L at $80^{\circ} \mathrm{C}$ for 5 days in aqueous buffered solutions at $\mathrm{pH}$ values of 2.2, 7.0, and 8.5. No detectable loss of gaba-L or appearance of gabapentin was observed in solution or solid-state. Therefore, the decrease in lactamization rate of gabapentin under high humidity conditions could not be explained by mass law effect because the reaction does

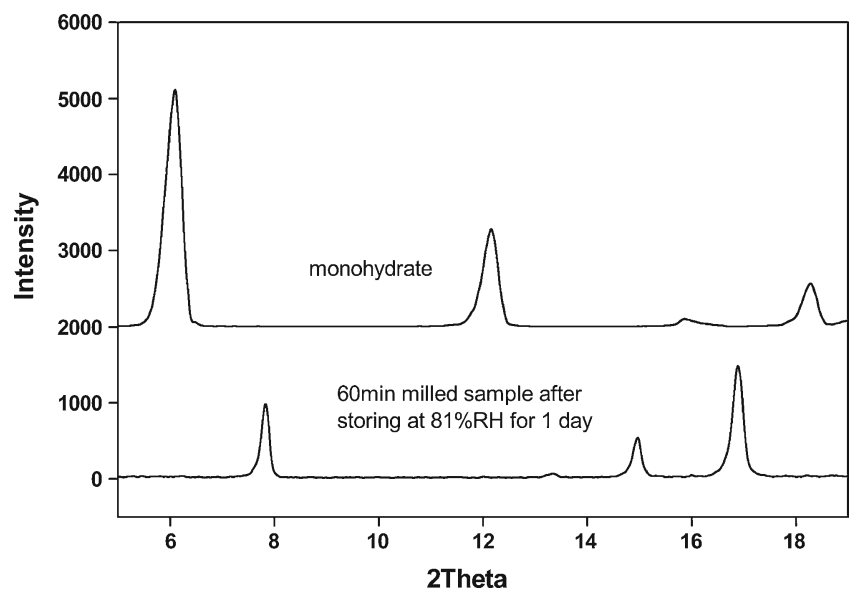

Fig. 6. XRD patterns of moisture treated milled gabapentin sample and gabapentin monohydrate not appear to be reversible even under these more rigorous conditions. It should be noted parenthetically that several patents (22-24) have disclosed a synthetic process for gabapentin involving the hydrolysis of gaba-L in the presence of $50 \%$ hydrochloric acid by refluxing at $108^{\circ} \mathrm{C}$ for $6 \mathrm{~h}$.

Formation of stable hydrate form at high humidity is another possible reason. An aliquot of gabapentin sample was milled for 60 min to generate high level of crystal defects and then the sample was stored at $81 \% \mathrm{RH}$ and room temperature for 1 day. From the XRD pattern, there was no detectable gabapentin hydrate peaks (Fig. 6) found, but the lactamization rate was significantly decreased. Therefore, formation of stable hydrate form is not likely the cause for the stabilizing effect.

Another explanation for the stabilizing effect of moisture on the chemical degradation of gabapentin is that high humidity conditions facilitate the "self-healing" of crystal defects generated by milling. Thus at high humidity, milling-damaged crystals are able of recovering or at least transitioning to a form of gabapentin that is incapable of spontaneous dehydration. And if the recovery (or termination) process is kinetically competitive with lactamization then a decrease in the apparent lactamization rate will be observed.

Although altered crystalline forms due to manufacturing stress are consistent with the changes observed in PXRD and ssNMR data and with the evaluation of the relationships between surface area and lactamization rate for milled and unmilled gabapentin, the direct detection of specific form transitions was not observed and therefore recovery or "self-

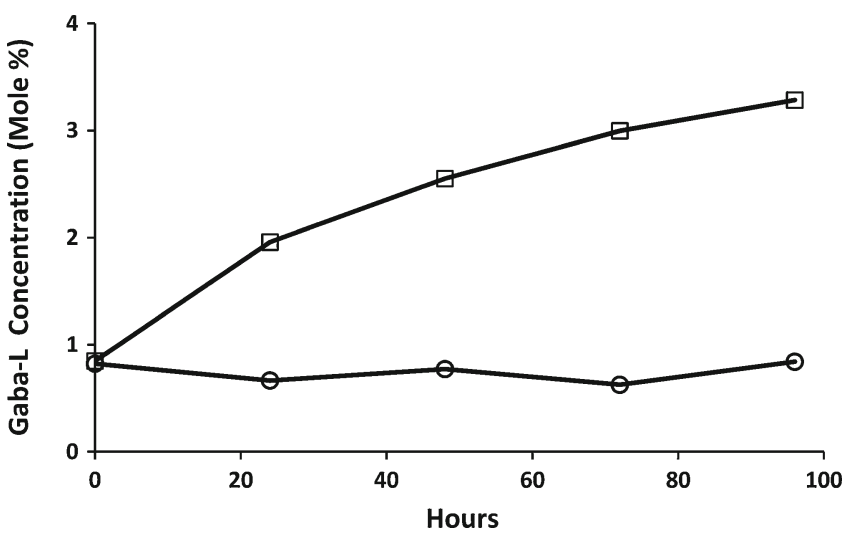

Fig. 7. Gaba-L formation from moisture pretreated gabapentin sample. Empty square sample was stored at $25^{\circ} \mathrm{C} 0 \% \mathrm{RH}$ for $24 \mathrm{~h}$ before thermal stress, empty circle sample was stored at $25^{\circ} \mathrm{C} 81 \%$ $\mathrm{RH}$ for $24 \mathrm{~h}$ before thermal stress 
healing" could not be directly observed. This inability to obtain direct empirical evidence was primarily due to the detection limits of the physical characterization methods employed and the small extent of milling-induced form changes under the conditions used in this study. Subsequent characterization efforts using additional ssNMR methods (e.g., relaxation times) and PXRD after much more rigorous milling are presented in future manuscripts. These studies showed additional evidence of physical transformation during milling (as has been previously reported in the literature (7)) and evidence of accelerated reformation of the stable polymorphic form (form II) upon exposure to high humidity.

Our hypothesis that humidity promotes the recovery of milling-induced crystal disorder predicts that milled gabapentin subjected to high humidity for a short period of time and then subjected to thermal stress under dry conditions would show significantly decreased rates of lactamization when compared to a control sample of milled gabapentin that had not been exposed to high humidity. This experiment was conducted and the results are presented in Fig. 7. The lactamization rate for the aliquot that was exposed to $81 \% \mathrm{RH}$ for $24 \mathrm{~h}$ prior to being exposed to dry heat for an additional $72 \mathrm{~h}$ was much lower than the control sample which was not exposed to high humidity. This result is consistent with the hypothesis that high atmosphere moisture facilitates the recovery of manufacturing-induced crystal defects thereby stabilizing gabapentin. Moreover these results are also inconsistent with gaba-L vaporization or mass law effect hypotheses both of which would have predicted comparable rates of lactamization for the humidified and control samples.

\section{CONCLUSION}

Gabapentin has been shown to be susceptible to millinginduced chemical instability that can be attributed to the formation of crystal defects that lead to increased rates of lactamization. Moreover, these defects can be annealed by exposure to high atmospheric moisture which stabilizes the milling-damaged drug substance. Additional studies are underway to develop quantitative solid-state degradation model that incorporates the effects of manufacturing (crystal damage and in-process lactamization) with the effects of comilled excipients and the environment effects of storage (humidity and temperature) on the kinetics of lactamization. The overarching research objective is to use gabapentin as a prototype drug substance to investigate the relationship between manufacturing variation and drug stability.

\section{REFERENCES}

1. Ibers JA. Gabapentin and gabapentin monohydrate. Acta Crystallogr, Section C: Cryst Struct Commun. 2001;C57:641-3.

2. Pesachovich M, Singer C, Pilarski G. Preparation of gabapentin. WO98/28255, 25 pp. 1998
3. Reece HA, Levendis DC. Polymorphs of gabapentin. Acta Crystallogr, Section C: Cryst Struct Commun. 2008;C64(3): o105-8.

4. Kearney AS, Mehta SC, Radebaugh GW. The effect of cyclodextrins on the rate of intramolecular lactamization of gabapentin in aqueous solution. Int J Pharm. 1992;78(1):25-34.

5. Hsu CH, Ke WT, Lin SY. Progressive steps of polymorphic transformation of gabapentin polymorphs studied by hot-stage FTIR microspectroscopy. J Pharm Pharmaceut Sci. 2010;13:67-77.

6. Brago D, Grepioni F, Maini L, Rubini K, Polito M, Brescello R, et al. Polymorphic gabapentin: thermal behavior, reactivity and interconversion of form in solution and solid-state. New J Chem. 2008;32:1788-95.

7. Lin SY, Hsu CH, Ke W. Solid-state transformation of different gabapentin polymorphs upon milling and co-milling. Int J Pharm. 2010;396:83-90.

8. Zour E, Lodhi SA, Radebaugh GW. Stability studies of gabapentin in aqueous solutions. Pharm Res. 1992;9(5):595-600.

9. Zambon E, Giovanetti R, Cotarca L, Pasquato L. Mechanistic investigation on 2-aza-spiro[4,5]decan-3-one formation from 1(aminomethyl)cyclohexylacetic acid (gabapentin). Tetrahedron. 2008;64(28):6739-43.

10. Cutrignelli A, Denora N, Lepedota A, Trapani A, Laquintana $\mathrm{V}$, Latrofa $\mathrm{A}$, et al. Comparative effects of some hydrophilic excipients on the rate of gabapentin and baclofen lactamization in lyophilized formulations. Int J Pharm. 2007;332(12):98-106.

11. Chawla M, Raghuvanshi R, Rampal A. Sustained release oral tablets of gabapentin and process for their preparation. WO 2005/020978 A1, 23 pp. 2005

12. Augart H, Gebhardt U, Herrmann W. Lactam-free amino acids. US $6,054,482,5$ pp. 2000

13. Singer C, Pilarski F, Pesachovich M. Stable gabapentin containing more than $20 \mathrm{ppm}$ of chlorine ion. WO 01/97612 A1, 24 pp. 2001

14. Manikandan R, Gogia A, Roy S, Makik R. Gabapentin tablets and methods for their preparation. WO 2004/032905 A1, 17 pp. 2004

15. Sherman B. Solid compositions comprising gabapentin having improved stability. WO 2004/014356, 10 pp. 2004

16. Chawla M, Raghuvanshi R, Rampla A. Stable sustained-release oral dosage forms of gabapentin and process for preparation thereof. WO 2005/077332 A2, 20 pp. 2005

17. Yande V, Kulkarni S, Narasimharaghavan S, Meenakshisunderam S. Stable liquid formulation of antiepileptic agents. WO 2007/ 107835 A2, 9 pp. 2007

18. Lloret P. Solid pharmaceutical composition of gabapentin. WO 2007/128495, 25 pp. 2007

19. Volpe DA, Gupta A, Ciavarella AB, Faustino PJ, Sayeed VA, Khan MA. Comparison of the stability of split and intact gabapentin tablets. Int J Pharm. 2008;350(1-2):65-9.

20. Gupta A, Ciavarella AB, Sayeed VA, Khan MA, Faustino PJ. Development and application of a validated HPLC method for the analysis of dissolution samples of gabapentin drug products. J Pharm Biomed Anal. 2008;46(1):181-6.

21. Steiner K, Herrmann W, Baumagarten Z, Crone G. Process for the preparation of cyclic amino acids and intermediates useful in the process. US 5068413, 8 pp. 1991

22. Inman EL, Tenbarge HJ. High-low chromatography: estimating impurities in HPLC using a pair of sample injections. J Chromatogr Sci. 1988;26:89-94.

23. Geibel W, Hartenstein J, Herrmann W, Witzke J. Process for the preparation of 1-aminomethyl-1-cyclohexaneacetic acid. US 5091567, 12 pp. 1992

24. Bryans J, Morrell A. Novel stereoselective processes for the preparation of gabapentin analogues. WO 9914184, 19 pp. 1999 